Acta Crystallographica Section D

Biological

Crystallography

ISSN 0907-4449

James F. Parsons, ${ }^{\mathrm{a} *}$ Katherine $\mathbf{M}$. Shi $^{\mathrm{a}}$ and Jane E. Ladner ${ }^{\mathrm{a}, \mathrm{b}}$

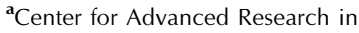
Biotechnology, University of Maryland Biotechnology Institute, USA, and ${ }^{\mathbf{b}}$ National Institute of Standards and Technology, USA

Correspondence e-mail: parsonsj@umbi.umd.edu

Received 23 January 2008

Accepted 26 February 2008

PDB References: apo-MenF, 3bzm, r3bzmsf; MenF-Mg ${ }^{2+}$ complex, 3bzn, r3bznsf.

\section{Structure of isochorismate synthase in complex with magnesium}

The electron carrier menaquinone is one of many important bacterial metabolites that are derived from the key intermediate chorismic acid. MenF, the first enzyme in the menaquinone pathway, catalyzes the isomerization of chorismate to isochorismate. Here, an improved structure of MenF in a new crystal form is presented. The structure, solved at $2.0 \AA$ resolution in complex with magnesium, reveals a well defined closed active site. Existing evidence suggests that the mechanism of the reaction catalyzed by MenF involves nucleophilic attack of a water molecule on the chorismate ring. The structure reveals a well defined water molecule located in an appropriate position for activation by Lys190 and attack on the substrate.

\section{Introduction}

The menaquinone-specific isochorismate synthase (MenF) from Escherichia coli is a chorismate-utilizing enzyme that catalyzes the formation of isochorismate for use in menaquinone biosynthesis (Fig. 1; Daruwala et al., 1997). Menaquinone is an electron carrier that is essential for anaerobic electron transport in bacteria. Isochorismate is also an intermediate in the biosynthesis of siderophores such as the iron chelator enterobactin. The E. coli genome encodes a second isochorismate synthase, EntC, which is involved in this pathway (Buss et al., 2001). The unstable nature of isochorismate requires that its formation and incorporation into different products be tightly regulated (DeClue et al., 2006). Indeed, all aspects of chorismate metabolism are highly regulated since it occupies a key metabolic branchpoint (Dosselaere \& Vanderleyden, 2001).

Three of the most important chorismate-utilizing enzymes, isochorismate synthase, anthranilate synthase (AS) and aminodeoxychorismate synthase, share a common ancestor and a common catalytic mechanism (Kolappan et al., 2007). He and coworkers showed that catalysis was initiated in each case by nucleophilic attack at $\mathrm{C} 2$ of chorismate (He et al., 2004) by either water or ammonia. In the case of isochorismate synthase, the nucleophile is a water molecule. In addition to these central metabolic enzymes, several secondary metabolic enzymes including salicylate synthase (SS) and aminodeoxyisochorismate synthase (PhzE) also share this mechanistic similarity.

Recently, the structure of $E$. coli MenF was solved at a resolution of $2.5 \AA$. The structure and associated biochemical experiments revealed that Lys190 is the catalytic base that activates a water molecule for attack at C2 of chorismate (Kolappan et al., 2007). However, owing to the manner in which MenF crystallized, the structure was less than ideal in terms of revealing many of the details<smiles>C=C(OC1C=C(C(=O)O)C=CC1O)C(=O)O</smiles><smiles>C=C(OC1C=CC=C(C(=O)O)C1O)C(=O)O</smiles>

(C) 2008 International Union of Crystallography Printed in Singapore - all rights reserved
Figure 1

Reaction catalyzed by MenF. 
relevant to ligand binding and catalysis. The active site was in a disorganized 'open' conformation, the $B$ factors for the entire structure were fairly high and many atoms were not present in the density, including some key elements of the active site.

Here, we report two new crystal forms of MenF. The first reveals a structure in which MenF is in an open conformation similar to that seen in the structure determined by Kolappan and coworkers. The second crystal form of MenF, however, was solved in complex with magnesium, a metal that is required for catalytic activity. This structure reveals a closed and well organized active site with a sulfate ion occupying the chorismate-binding site and mimicking the interaction of the $\mathrm{C} 1$ carboxylate of chorismate with the metal ion. Additionally, an ordered water molecule is observed in a position favorable for activation by Lys190, the putative catalytic base.

\section{Experimental}

\subsection{Cloning and expression of the isochorismate synthase MenF}

The men $F$ gene from $E$. coli was cloned from $E$. coli genomic DNA (Sigma) ${ }^{\mathbf{1}}$ by PCR using KOD polymerase (Novagen) and synthetic primers (Integrated DNA Technologies) compatible with the menF sequence. The amplified men $F$ fragment was inserted into the vector pET15b (Novagen) for protein expression. E. coli strain BL21 (DE3) was used to express the MenF enzyme. Cells transformed with the pET15b-MenF plasmid were grown in ZYP-5052 medium containing $200 \mu \mathrm{g} \mathrm{ml}^{-1}$ ampicillin. Initial growth was at $310 \mathrm{~K}$. When the culture density reached an optical density of $\sim 0.2$ at $600 \mathrm{~nm}$, the temperature was reduced to $293 \mathrm{~K}$ and the cells were harvested by centrifugation after $\sim 15 \mathrm{~h}$. Cells were lysed by sonication and His-tagged MenF was purified by $\mathrm{Co}^{2+}$-ion affinity chromatography as directed by the resin manufacturer (Sigma). Human $\alpha$-thrombin (Haematologic Technologies) was used to remove the His tag from the purified protein. Careful monitoring of the digestion by mass spectrometry was required since thrombin was found to cleave MenF at a second discrete site, cutting the protein roughly in half. This was largely avoided by limiting the time that the digestion was allowed to proceed. Thrombin was removed using benzamidine agarose and MenF was further purified by passage over a second $\mathrm{Co}^{2+}$ column. Pure MenF was dialyzed against $50 \mathrm{~m} M$ Tris, $5 \mathrm{mM} \mathrm{MgCl}, 1 \mathrm{~m} M$ DTT pH 7.6, concentrated to $\sim 20 \mathrm{mg} \mathrm{ml}^{-1}$ and stored at $193 \mathrm{~K}$ in $250 \mu \mathrm{l}$ aliquots.

\subsection{Crystallization}

MenF crystallizes in at least three distinct forms. Crystals of apoMenF (Table 1) were grown at $\sim 295 \mathrm{~K}$ via sitting-drop vapor diffusion by mixing equal volumes of protein solution, as described above, with a well solution containing $0.2 \mathrm{M}$ trisodium citrate, $6 \%$ glycerol, $8 \%$ PEG 3350 and $5 \mathrm{mM}$ chorismic acid. Apo-MenF crystals appeared in 1-2 d as trigonal bipyramidal blocks that ultimately grew as large as $1.0 \mathrm{~mm}$ on each edge. Crystal growth appeared to depend on the presence of chorismate even though it was not found in the density map. Despite their impressive size and visual appearance, these crystals only yielded diffraction to $2.0 \AA$ resolution. Crystals of the MenF- $\mathrm{Mg}^{2+}$ complex (Table 1) were grown at $\sim 295 \mathrm{~K}$ via hanging-drop vapor diffusion by mixing equal volumes of protein

\footnotetext{
$\overline{1}$ Certain commercial materials, instruments and equipment are identified in this manuscript in order to specify the experimental procedure as completely as possible. In no case does such identification imply a recommendation or endorsement by the National Institute of Standards and Technology, nor does it imply that the material, instrument or equipment identified is the best available for the purpose.
}

Table 1

Data-collection and refinement statistics.

\begin{tabular}{lll}
\hline & Apo-MenF & MenF- $\mathrm{Mg}^{2+}$ complex \\
\hline PDB code & 3 bzm & 3 bzn \\
Space group & $P 3_{2} 21$ & $P 2_{1} 2_{1} 2_{1}$ \\
Unit-cell parameters $(\AA)$ & $a=b=72.4$, & $a=65.3, b=70.5$, \\
& \multicolumn{1}{c}{$c=164.0$} & \multicolumn{1}{c}{$c=90.3$} \\
No. of measured intensities & 289609 & 85731 \\
No. of unique reflections & 36355 & 28882 \\
Resolution of data $(\AA)$ & $19.67-1.95$ & $29.62-2.00$ \\
$R_{\text {merge }}$ & $0.064(0.474)$ & $0.074(0.322)$ \\
Completeness $(\%)$ & $98.1(84.8)$ & $99.2(95.7)$ \\
Redundancy & $7.97(4.61)$ & $2.97(2.82)$ \\
Mean $I / \sigma(I)$ & $14.7(2.6)$ & $9.2(3.2)$ \\
Refinement statistics & $19.69-1.95$ & $20.00-2.00$ \\
Resolution limits & $0.245(0.349)$ & $0.202(0.298)$ \\
$R$ & $0.308(0.398)$ & $0.284(0.374)$ \\
$R_{\text {free }}$ & 104 & 218 \\
No. of water molecules & 0.020 & 0.021 \\
R.m.s.d. bond length $(\AA)$ & 92.0 & 94.7 \\
Ramachandran plot, preferred $(\%)$ & 6.6 & 5.3 \\
Ramachandran plot, allowed $(\%)$ & 1.4 & 0.0 \\
Ramachandran plot, outliers $(\%)$ & & 28.3 \\
Average $B$ factor $\left(\AA^{2}\right)$ & 47.9 & 29.5 \\
$\quad$ Main chain & 49.4 & 30.0 \\
$\quad$ Side chain & 44.9 & \\
$\quad$ Water & & \\
\hline
\end{tabular}

solution that had been dialyzed against $50 \mathrm{~m} M$ Tris pH 7.8, $50 \mathrm{~m} M$ $\mathrm{MgSO}_{4}$ with a well solution containing $0.1 \mathrm{M}$ bis-tris $\mathrm{pH} 6.5,20 \%$ PEG MME 5000. These crystals also appeared in 1-2 d, but tended to be small plates $(\sim 0.1 \mathrm{~mm}$ in the largest dimension) that nonetheless also diffracted to $2.0 \AA$ resolution. A third crystal form of MenF has previously been reported, apparently using protein that had an intact histidine-affinity tag (Kolappan et al., 2007).

\subsection{Data collection}

Diffraction data for the open and closed structures were collected using a Rigaku MicroMax-007 rotating-anode generator with an R-AXIS IV ${ }^{++}$detector (Rigaku/MSC, The Woodlands, Texas, USA). The crystals were cooled to $105 \mathrm{~K}$ using an X-stream 2000 Cryocooler (Rigaku/MSC, The Woodlands, Texas, USA). The closed form crystals were cryoprotected by dipping the crystal in a drop made up of equal volumes of well solution and 50\% PEG MME 5K before mounting it on the goniometer. The open-form crystals did not require additional cryoprotection. Diffraction data were processed with CrystalClear/d*TREK (Pflugrath, 1999). Statistics are shown in Table 1.

\subsection{Refinement}

For the closed form, the Matthews coefficient $\left(2.10 \AA^{3} \mathrm{Da}^{-1}\right)$ indicated that there was one molecule in the asymmetric unit. The structure was solved with the maximum-likelihood molecularreplacement program Phaser (McCoy et al., 2005) using the structure of PDB entry 2eua as a model. The model was viewed and adjusted and parts were rebuilt with Coot (Emsley \& Cowtan, 2004). REFMAC5 was used for refinement between viewing sessions (Murshudov et al., 1997). The final statistics are shown in Table 1. Phaser was used to place the closed-form molecule in the open-form data. Again, the Matthews coefficient $\left(2.89 \AA^{3} \mathrm{Da}^{-1}\right)$ indicated that there was only one molecule in the asymmetric unit. Coot and REFMAC5 were again used to adjust, view and refine the model. 


\section{Results and discussion}

\subsection{Comparison with existing MenF structure}

The structure of apo-MenF determined using data from the trigonal bipyramidal crystals was quite similar to the structure determined previously by Kolappan and coworkers despite the fact that the crystals were of a different form. Using the DaliLite (Holm \&
Park, 2000) analysis software, the two structures were superimposed and an r.m.s.d. of $0.9 \AA$ for all $\mathrm{C}^{\alpha}$ atoms was calculated. Like the Kolappan structure, no ligand or metal was observed in the active site. These structures represent an open conformation of MenF in which the active site is solvent-exposed and disorganized (Figs. $2 a$ and $2 b$ ). The $B$ factors determined for this conformation were also quite high, although fewer atoms are missing from the model, perhaps owing to



(a)
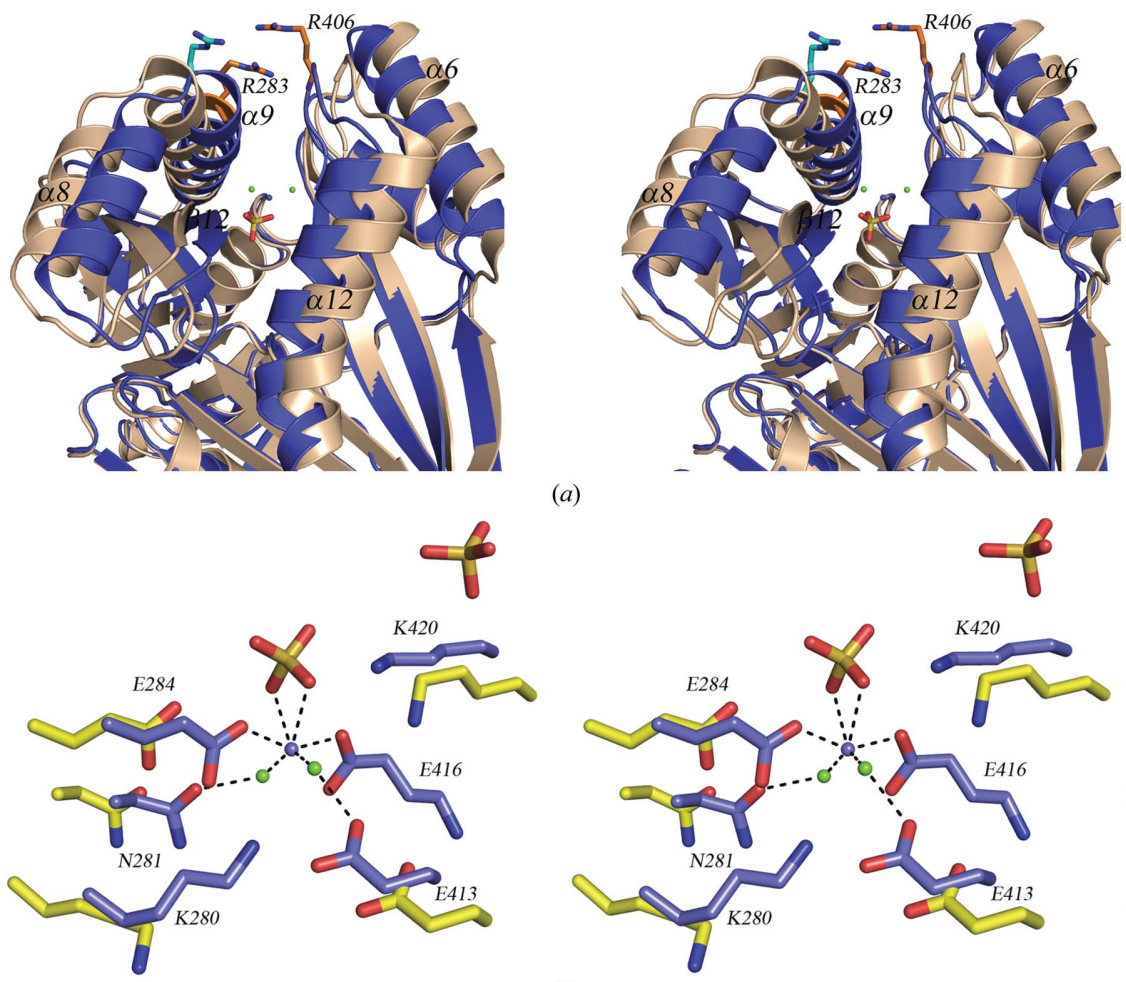

(b)
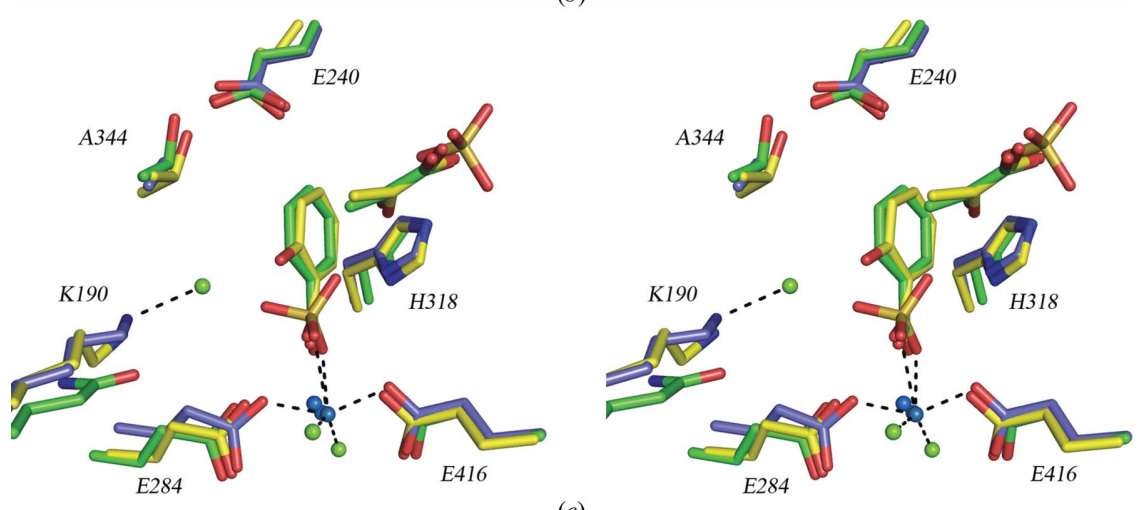

(c)

Figure 2

(a) Superposition of magnesium-bound MenF with apo-MenF (PDB code 2eua), illustrating the relative positions of the secondary-structural elements that define the active-site cleft of MenF in the presence and absence of magnesium. The MenF- $\mathrm{Mg}^{2+}$ complex is shown in blue; 2eua is shown in beige. The interaction of $\operatorname{Arg} 283$ with Arg406 is shown, along with the alternate conformation of Arg283 in apo-MenF. The loop containing Arg 406 is poorly defined by electron density in the apo-MenF structure. (b) Superposition of the active sites of MenF with bound magnesium (shown with blue $\mathrm{C}$ atoms; this work) and without magnesium (PDB code 2eua; shown in yellow), illustrating the differences in the conformations of key active-site residues in the presence and absence of magnesium. The side chain of Glu416 was not present in the 2eua structure. Water molecules coordinated to $\mathrm{Mg}^{2+}$ are shown as green spheres. (c) The remarkable conservation among active-site residues of chorismate-utilizing enzymes suggests factors beyond the first sphere of the active-site impact catalysis and influence product distribution. A superposition of MenF (blue $\mathrm{C}$ atoms), anthranilate synthase (PDB code 1i7q; green $\mathrm{C}$ atoms) in complex with benzoate and pyruvate and SS (PDB code 2fn1; yellow C atoms) in complex with salicylate and pyruvate is shown. Water molecules from MenF are shown in green; $\mathrm{Mg}^{2+}$ ions are shown in blue. The water molecule adjacent to Lys190 is in position to attack a substrate at $\mathrm{C} 2$ upon activation. Sulfate ions are found in locations analogous to those of the substrate carboxylate group and the cleaved pyruvoyl group in other chorismate-utilizing enzymes. the higher resolution (Table $1 ; B$-factor plots as well as Ramachandran plots are included in the supplementary material ${ }^{2}$ ).

The structure determined from the orthorhombic crystal form of MenF is markedly different and reveals a closed organized active site containing a magnesium ion, a metal required for catalysis, and two sulfate ions (Figs. 2 and 3). The average $B$ factors calculated for this structure were considerably lower than those for either the apo-MenF structure presented here or the Kolappan structure. We have not had success in obtaining a structure of MenF with chorismate or isochorismate bound. However, sulfate appears to mimic the interaction of the substrate carboxylate with the metal ion (Fig. 2c).

\subsection{Open versus closed conformation}

In the metal-bound form of MenF, a combination of gross movements of secondary-structural elements and a fine reorganization in the vicinity of the metal ion close off solvent access to the chorismate-binding site. Among the more significant shifts are the movements of four $\alpha$-helices $(\alpha 6, \alpha 8 \alpha 9$ and $\alpha 12)$ and two $\beta$-strands ( $\beta 12$ and $\beta 22$ ) towards the metal and each other, as well as the movement of the loop (residues 404-410) between $\beta 22$ and $\alpha 12$ towards $\alpha 9$ across the cleft leading to the active site. In particular, a stacking interaction between the guanidino groups of Arg283 and Arg406 appears to stabilize the closed magnesium-bound conformation. The magnesium ion is coordinated to two sulfate $\mathrm{O}$ atoms, two water molecules, and the carboxylate O atoms of Glu284 and Glu416 (Fig. 3b). Fig. 2(a) illustrates the open and closed conformations of MenF.

\subsection{Comparison with other chorismate-utilizing enzymes}

It has been well documented that MenF, AS, aminodeoxychorismate synthase and SS have very similar structures (Zwahlen et al., 2007). The active sites are in fact nearly identical in these enzymes. Fig. 2(c) illustrates the similarity between the active-site regions of MenF, AS and SS. Interestingly, the two active-site sulfate ions found in MenF are positioned such that they mimic the carboxylate groups of the substrate/

\footnotetext{
2 Supplementary materal has been deposited in the IUCr electronic archive (Reference: HM5059). Services for accessing this material are given at the back of the journal.
} 
product as well as that of the cleaved puruvoyl group seen in the other structures. Indeed, a key remaining mechanistic issue regarding catalysis by chorismate-utilizing enzymes is identification of the factors that control retention or elimination of pyruvate from the ring.

The structure of MenF reveals a single notable difference between the active sites of MenF and SS, an enzyme that eliminates pyruvate from an isochorismate intermediate. Ala344 of MenF corresponds to Thr348 of SS and is the only nonconserved first-sphere active-site residue. However, mutagenesis experiments were unrevealing (Kolappan et al., 2007) and no other evidence exists to suggest that this factor controls product distribution. It appears to be more likely that either a combination of subtle factors or an element of the protein outside the active site impacts the ultimate outcome of catalysis. For instance, differences in the binding affinity of isochorismate could impact catalysis. If the closed conformation of SS had enhanced stability compared with that of MenF, elimination of pyruvate could be favored. Something as simple as a key hydrogen bond or salt bridge across the active-site cleft could favor a reduced product off-rate in SS, for example, leading to efficient elimination of pyruvate.

A primary difference between the isochorismate-forming enzymes (MenF/EntC and salicylate synthase) and the homologs that aminate chorismate (anthranilate synthase, aminodeoxychorismate synthase and PhzE) is the residue corresponding to Lys190 of MenF. Anthranilate synthase and other enzymes that aminate chorismate typically have a glutamine or asparagine residue at this position in lieu of the lysine. Substantial biochemical evidence indicates that MenF uses this lysine as a catalytic base, activating a water molecule for attack at $\mathrm{C} 2$ of chorismate. Importantly, the structure of MenF presented here supports this notion, revealing a well defined water molecule located $2.5 \AA$ from the $\varepsilon$-amino group of Lys 190 and $\sim 3.0 \AA$ from the likely location of $\mathrm{C} 2$ of the substrate (Fig. $2 c$ ). The identity of this Lys or Gln/Asn residue, however, does not correlate with retention or elimination of pyruvate. Among the water-nucleophile group and the amine-nucleophile group there are enzymes that retain pyruvate and enzymes that eliminate it. Additional experimentation that targets second-sphere active-site residues may prove more revealing as to the fate of the pyruvoyl group.

This work was supported in part by a grant from the National Institute of Allergy and Infectious Diseases (AI067530) to JFP.

Figure 3

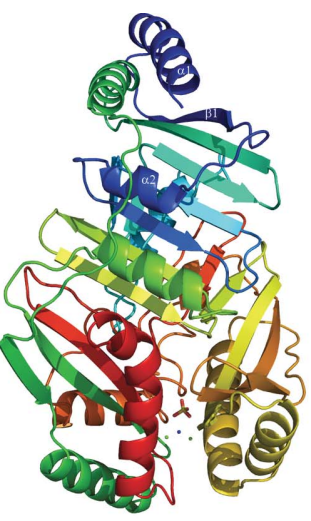

(a)
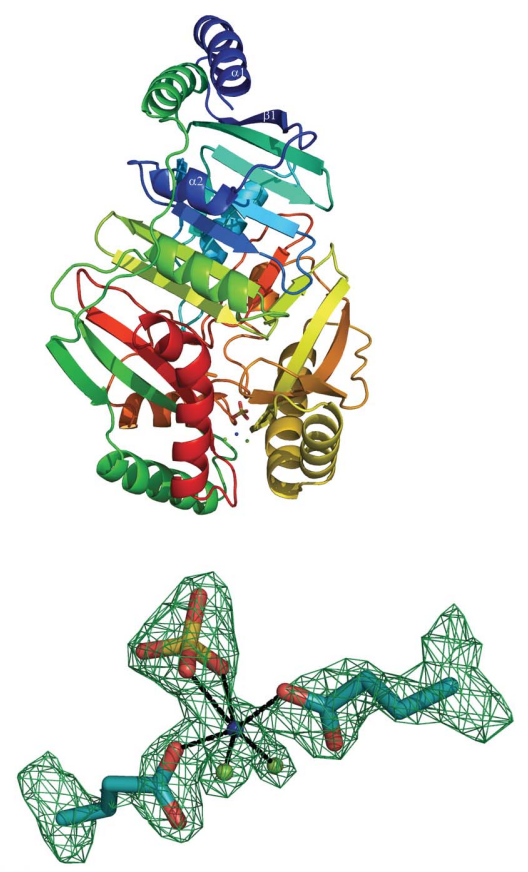

(b)

Improved structure of the isochorismate synthase MenF. (a) Stereoview of the MenF monomer colored with two water molecules and a sulfate ion. (b) Magnesium coordination in the active site of MenF. The positive difference density calculated after omitting Glu284, Glu416, the sulfate ion, $\mathrm{Mg}^{2+}$ and its associated two O atoms from a round of refinement with REFMAC5 (Murshudov et al., 1997) is contoured at $4 \sigma$ and shown with the refined model.

\section{References}

Buss, K., Muller, R., Dahm, C., Gaitatzis, N., Skrzypczak-Pietraszek, E., Lohmann, S., Gassen, M. \& Leistner, E. (2001). Biochim. Biophys. Acta, 1522, 151-157.

Daruwala, R., Bhattacharyya, D. K., Kwon, O. \& Meganathan, R. (1997). J. Bacteriol. 179, 3133-3138.

DeClue, M. S., Baldridge, K. K., Kast, P. \& Hilvert, D. (2006). J. Am. Chem. Soc. 128, 2043-2051.

Dosselaere, F. \& Vanderleyden, J. (2001). Crit. Rev. Microbiol. 27, 75-131.

Emsley, P. \& Cowtan, K. (2004). Acta Cryst. D60, 2126-2132.

He, Z., Stigers Lavoie, K. D., Bartlett, P. A. \& Toney, M. D. (2004). J. Am. Chem. Soc. 126, 2378-2385.

Holm, L. \& Park, J. (2000). Bioinformatics, 16, 566-567.

Kolappan, S., Zwahlen, J., Zhou, R., Truglio, J. J., Tonge, P. J. \& Kisker, C. (2007). Biochemistry, 46, 946-953.

McCoy, A. J., Grosse-Kunstleve, R. W., Storoni, L. C. \& Read, R. J. (2005). Acta Cryst. D61, 458-464.

Murshudov, G. N., Vagin, A. A. \& Dodson, E. J. (1997). Acta Cryst. D53, 240-255.

Pflugrath, J. W. (1999). Acta Cryst. D55, 1718-1725.

Zwahlen, J., Kolappan, S., Zhou, R., Kisker, C. \& Tonge, P. J. (2007). Biochemistry, 46, 954-964. 